Received: November 25, 2017

Revision received: March 25, 2018

\title{
Dance Posture Analysis Based on Virtual Reality Technology and Its Application in Dance Teaching*
}

\author{
Xue Yang ${ }^{1}$ \\ Huaiyin Normal University
}

\author{
Yin Lyu ${ }^{2}$ \\ Huaiyin Normal University
}

\begin{abstract}
This paper proposes a dance posture analysis method based on eigenvector matching, and sets up a motion capture system that calculates motion parameters by similarity matching of characteristic planes. Considering the complexity of dance learning, the proposed system was adopted to acquire various postures of dance learners, and contrast their dance postures against the standard ones. The proposed analysis method was verified through a contrastive experiment, shedding new light on the innovation of digital dance teaching.
\end{abstract}

\section{Keywords}

Dance Teaching • Posture Analysis $\bullet$ Motion Capture • Virtual Reality • Similarity Matching

\footnotetext{
* This work is supported by China Education Information Technology Research 2018 Youth Project (186140095): Traditional Dance Movement Collection and Teaching Resource Library Construction Based on Motion Capture

${ }^{1}$ Correspondence to: Xue Yang (MA), Huaiyin Normal University, Huaian 223300, China. Email: lutaibai1981@ gmail.com ${ }^{2}$ Huaiyin Normal University, Huaian 223300, China. Email: 8201711037@ hytc.edu.cn
}

Citation: Yang, X., Lyu, Y., Dance Posture Analysis Based on Virtual Reality Technology and Its Application in Dance Teaching. Educational Sciences: Theory \& Practice, 18(5), 1224-1235. http://dx.doi.org/10.12738/estp.2018.5.022 
Thanks to the development of computer vision, virtual reality and assistant teaching have been closely merged to improve the efficiency and effect of teaching (Yang, Chen \& Ming, 2010), making digital teaching a reality. Computer vision refers to the identification of motions in the digital signals converted from the videos or images on the external environment, which are captured by one or more cameras and loaded into computer. The conversion, processing and analysis of the information are completed on computer (Moeslund \& Granum, 2001).

As the key to computer vision, motion analysis will enable students to discover irregular dance postures in time, if applied to dance teaching. The parameters of dance postures can be transformed into 3D motion models using the motion capture technique, and a database of teaching samples can be created for accurate evaluation of the teaching effect.

The past two decades has witnessed a rapid development of motion capture technology. The related studies mainly fall into the following categories: the strategy and application of motion capture, the data analysis of real-time motion, and the discussion on motion posture. One of the main applications of motion capture is dance art, an expression of many intangible cultural heritages.

Now, it is a fashionable way to employ motion capture technology for the digital protection of dance art. For instance, Qiu and Li, (2009) relied on Vicon MX hardware system to collect digital data on various postures of Chinese national dance, and set up a national dance posture database. Windsor (2004) used optimal motion capture device for the digital protection of puppet show. Lv and Guo (2011) digitally protected the musical dance performances in Confucius memorial ceremony. Shinoda et al. (2012) developed a visualization system for human motions and centre of gravity, which can easily classify different types of dances. Figure 1 illustrates how the system captures the motion of fan dance.

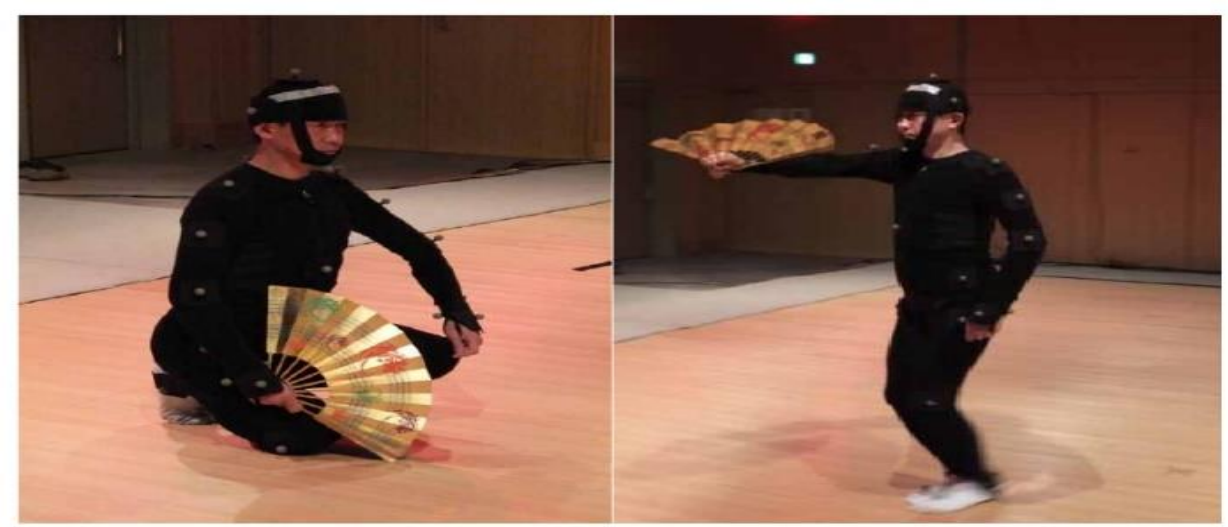

Figure 1. Motion capture of fan dance

The data acquired by motion capture devices are usually in discrete form. To disclose the law of posture change, the parameters of posture data need to be extracted from the discrete data through similarity analysis. Currently, posture analysis is based on either simulated annealing particle swarm optimization (SA-PSO) algorithm or human body model. 


\section{SA-PSO-based posture analysis}

After extracting posture parameters from the video on human arm motions, Renna, Chellali \& Achard (2011) calculated the conditional density by particle filter algorithm, treated it as the weight of the sampling point, and randomly updated the weight by Monte-Carlo method. Li, Sun \& Che (2012) introduced the SA-PSO to the posture analysis of human motions in three steps: Firstly, the 3D parameters were subjected to dimensionality reduction through principal component analysis (PCA),yielding a 2D compact posture space; Secondly, a fitness function was constructed on the human silhouette features in the low-dimensional space to measure the similarity between the model and image features; finally, the SA-PSO algorithm was adopted to enhance the analysis efficiency.

$$
\mathrm{E}\left(y_{t}, x_{t}\right)=S \times\left((1-\beta) \frac{B_{t}}{B_{t}+Y_{t}}+\beta \frac{R_{t}}{R_{t}+Y_{t}}\right)
$$

where $R_{t}$ is the area of human silhouette in the target image; $B_{t}$ is the projected area of the model; $Y_{t}$ is the overlap area of the two areas.

\section{Model-based posture analysis}

The first step of the model-based analysis is to build a model for human body. Then, the model is mapped to a $2 \mathrm{D}$ image, such that the similarity between the model and the image can be measured by an evaluation function. The quality of the model is critical to the analysis effect. References represent the skeleton of human body as a stick graph (Chen \& Lee, 2015; Bharatkumar, Daigle, Pandy \& Cai, 1994). Through least squares curve fitting, Zhao, Dai, Zhao \& Yuan (2012) approximated the law of human motion s with the polynomial coefficient: Firstly, the function relation $f(\mathrm{t})=a_{0} t+a_{1}$ of the coordinates for discrete data points $x_{i}, y_{i}$ and $z_{i}$ and time was calculated to disclose the general trend; Secondly, the most suitable function $f(\mathrm{t})$ was searched for in the coefficient space $\varnothing=\operatorname{span}\left\{\varphi_{0} t, \varphi_{1} t, \ldots, \varphi_{n} t\right\}$ :

$$
\mathrm{s}(\mathrm{t})=a_{0} \varphi_{0} t+a_{1} \varphi_{1} t+\cdots+a_{n} \varphi_{n} t
$$

Thirdly, the closest function $s(\mathrm{t})$ was determined as the most suitable function $f(\mathrm{t})$.

\section{Basic theory of motion capture technology}

The parameters for motion capture are mostly processed by computer. Tracking devices with special markers should be fixed at key positions of the moving target, and used to record the motions of the target. Then, 3D spatial parameters can be obtained via computer processing of the record, before going through the matching against the skeleton model.

In 1915, Fisher opened up the field of motion capture with the invention of Rotoscope. After decades of development in computer hardware, the existing motion capture devices generally consist of the following parts:

(1) Sensor: In motion capture system, sensor is a tracking device firmly attached to key parts of the moving target. This device can collect the physical signals on the displacement, velocity and angle of the moving object. The number of sensors depends on the specific site and object. Only a few sensors are needed to capture human 
motion, while many are necessary for the study of the movement of specific parts in human body, such as the change of facial expression, gestures, etc.

(2) Signal capturer: This device is seen only in some motion capture systems. The main function is to capture the position signals from the sensors. In popular optical motion capture systems, infrared HD cameras are installed to capture all sensor signals.

(3) Data transmitter: This device transmits all motion parameters from the signal capturer to the computer for timely analysis and processing. The transmission is implemented in an accurate and efficient manner.

(4) Data processor: This device processes and corrects the motion data and matches them with the 3D model, making the latter more realistic and natural.

As mentioned above, optical motion capture system enjoys immense popularity. This advanced system monitors and tracks the markers attached to the moving object with an array of highly sensitive cameras. In a typical optical motion capture system, there might be six to eight cameras arranged in specific places to shot the object from various angles. The cameras are often set to a fast shooting speed, sometimes as fast as 60 frames per second.

For better data acquisition, the subject is asked to wear single-colour clothes and attach markers, i.e. special reflective spheres, to the joints and other key parts of his/her body. During the capture process, the cameras track and detect the markers, and transfer the captured data to the computer. In the computer, the data is analysed, processed and saved in 3D form. Based on the 3D data, the spatial position of the moving target can be calculated for each motion, and the trajectory and other parameters of the subject can also be obtained.

The optical motion capture system boasts many advantages. For example, the subject is given enough space to move freely in multiple directions and angles, without being constrained by machines and wires. The high sampling frequency ensures the timely capture of every movement. On the downside, however, the optical motion capture system only works in a favourable environment: illumination and reflection are unwanted, the motions should better be captured indoor, the markers are easily occluded, and the cost and workload of postprocessing are rather high.

\section{Proposed optical motion capture system}

The author proposed an optical motion capture system with several near-infrared highly sensitive cameras and developed an S/D 3D space calibration algorithm to improve real-time 3D data. As shown in Figure 2, the proposed system mainly contains the following hardware devices: high-speed 3D data capture workstation, DIMS controller, special near-infrared highly sensitive cameras, highly sensitive markers, high precision 3D static and dynamic calibration lever. 


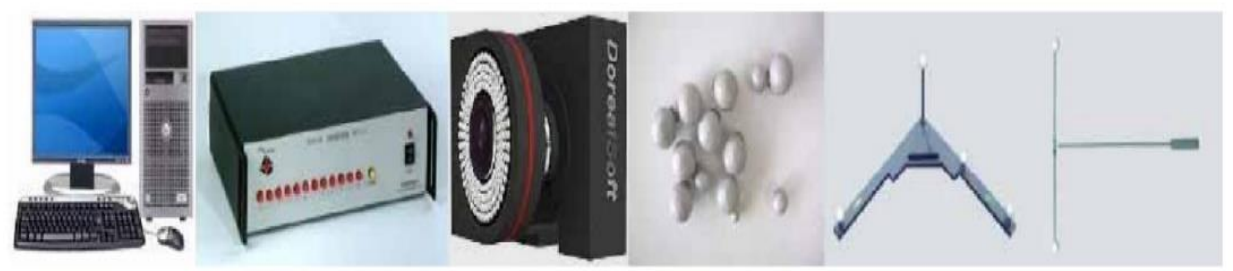

Figure 2. Hardware devices of the proposed system

The system collects data through non-contact near-infrared optical reflection. It supports the simultaneous shooting from 12 (expandable) angles. The number of markers is equal to or greater than 21.

The proposed system should be used in shady places indoor at low humidity and low ambient temperature. Besides, the working interface must be kept clean. The working environment is illustrated in Figure 3 below.
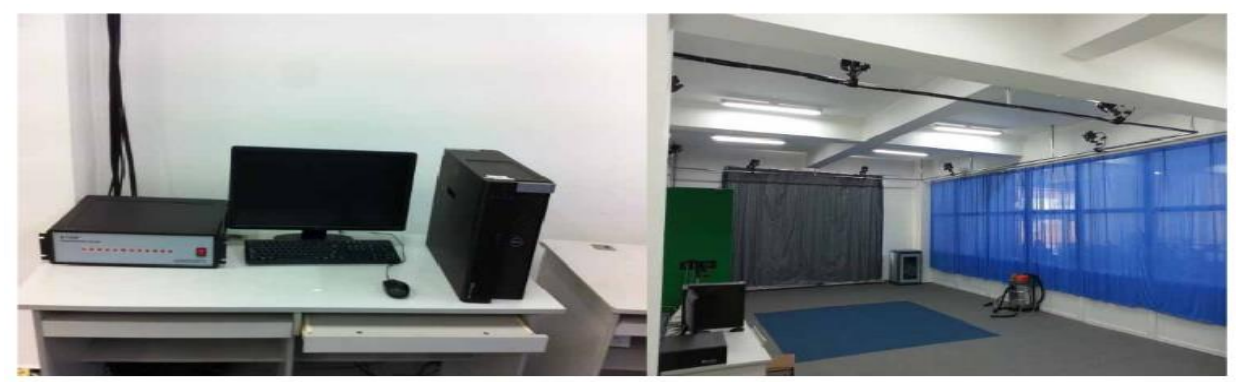

Figure 3. The working environment

Before the shooting, the subject should wear single-colour tight clothes and attach highly reflective markers to specific parts of the body, such as the head, chest and joints; the software to capture precise real-time 3D motion data should be initialized; the subject information, the shooting time and camera frequency should be configured; the cameras should be calibrated.

Once the shooting begins, the subject should complete the designed motions in the pre-set range. After the shooting, the system automatically switches to the $3 \mathrm{D}$ window to display the calculated 3D motion data (Figure $4)$.

\section{Dance Posture Analysis Based on Eigenvector Matching}

To realize efficient and accurate recognition of human postures, the author put forward an efficient posture analysis method based on similarity matching between characteristic planes. The proposed optical motion capture system was applied to collect real-time human motion data and extract the skeleton and its characteristic plane. Then, an efficient matching mechanism was established considering the plane eigenvector and its angle. Coupled with dance teaching, the analysis approach can provide stable and accurate parsing of body postures and identify the differences between body motions, laying a solid theoretical basis for scientific dance training. 


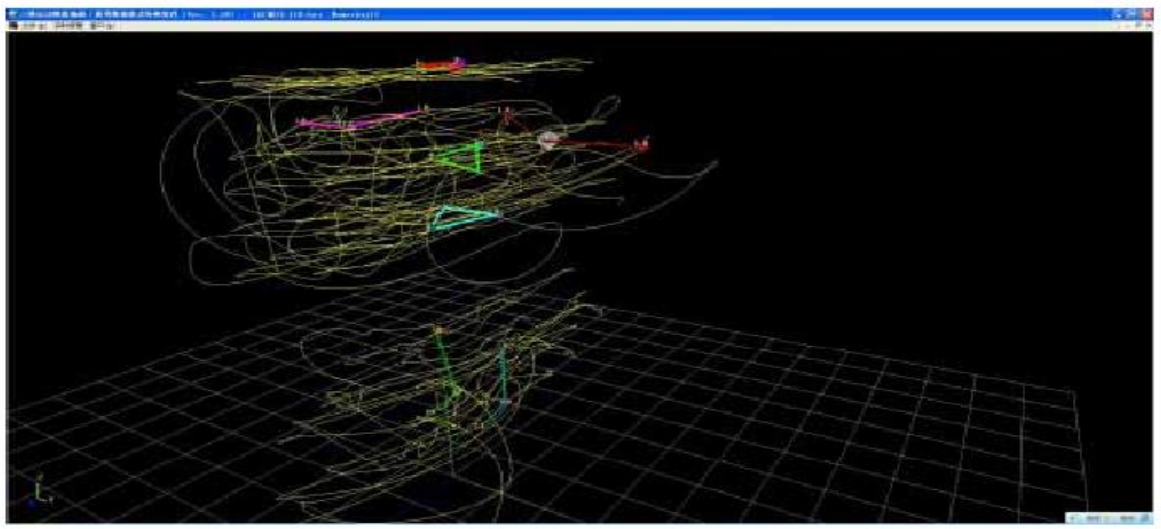

Figure 4. 3D data trajectories

\section{D data acquisition for motion capture}

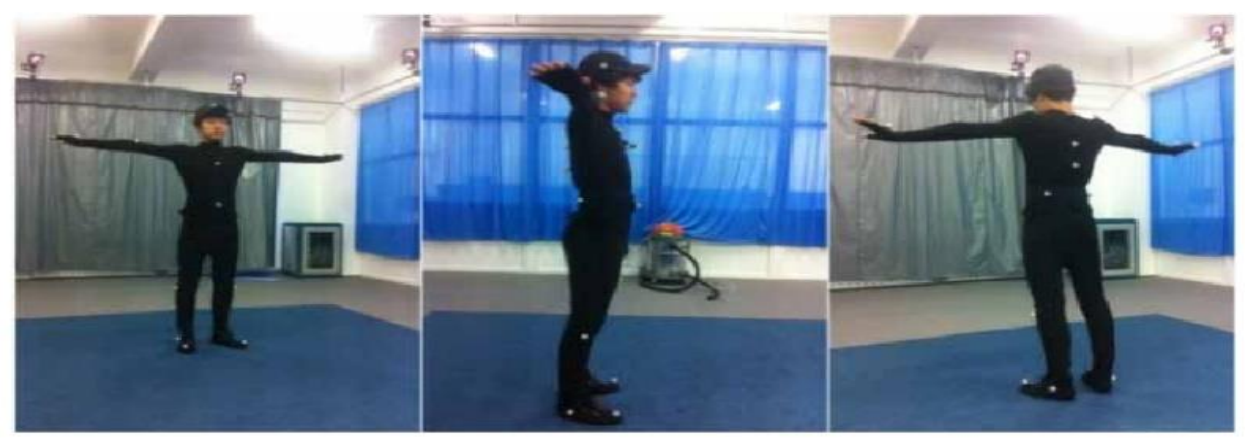

\section{Figure 5. Data acquisition}

During data acquisition, the subject wore single-colour clothes and carried 21 markers on the key parts of his/her body. After that, the subject stood in the pre-set range of motion space. Next, the high-precision 3D motion capture software was initialized, a specified time was configured, the specified dance postures were shot, and the movements of the markers were captured by the cameras. The entire process is displayed in Figure 5.

The human motion data captured from the markers were matched with the subject's model, forming a human motion posture database, which focuses on the postures of the head, body, hip and limbs. Here, the key points of dance posture (Figure 6) are used to mark the key parts of the human body. The lines between the key points represent the rigid body, whose shape does not change.

\section{Posture analysis based on eigenvector matching}

The posture analysis refers to the derivation of motion parameters through tracking, capturing, acquisition and analysis of posture features. If integrated to the teaching process, the motion analysis helps to make the 
teaching system more personalized and rational. The performance of the subject can be decomposed into details and every dance action can be demonstrated. The resulting parameters are conducive to the quantitative analysis of dance postures.

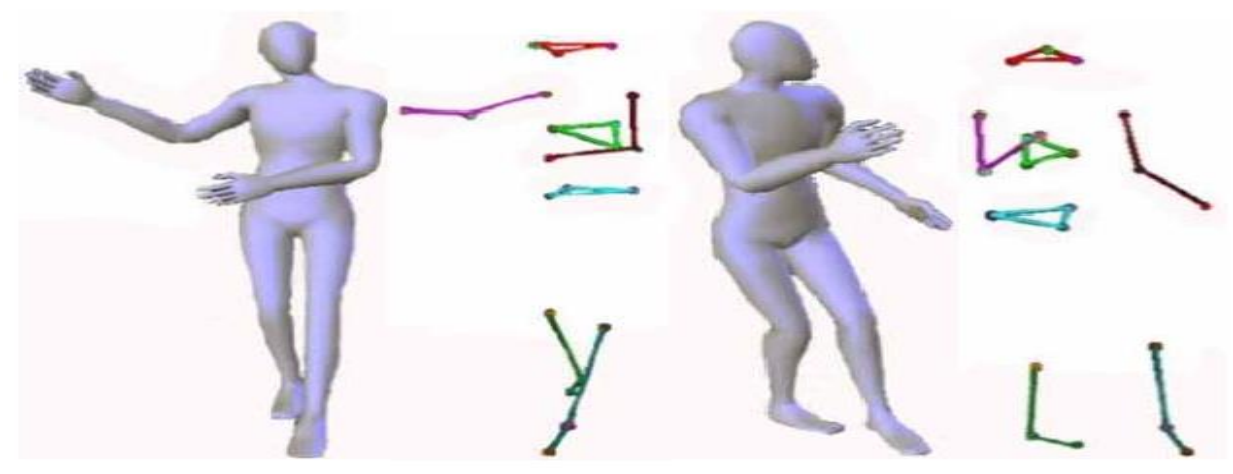

Figure 6. Key points of dance posture

The author proposed a human motion posture analysis method based on characteristic plane similarity matching, aiming to better analyse the motion state of the dancer. By this method, the traditional way to calculate the Euclidean distance based on multiple marking points is replaced with the calculation characteristic plane eigenvectors and their angles. The positions of the 21 markers were simplified as 7 characteristic planes for computing the difference and correlation between motions. The flow chart of the analysis method is provided in Figure 7.

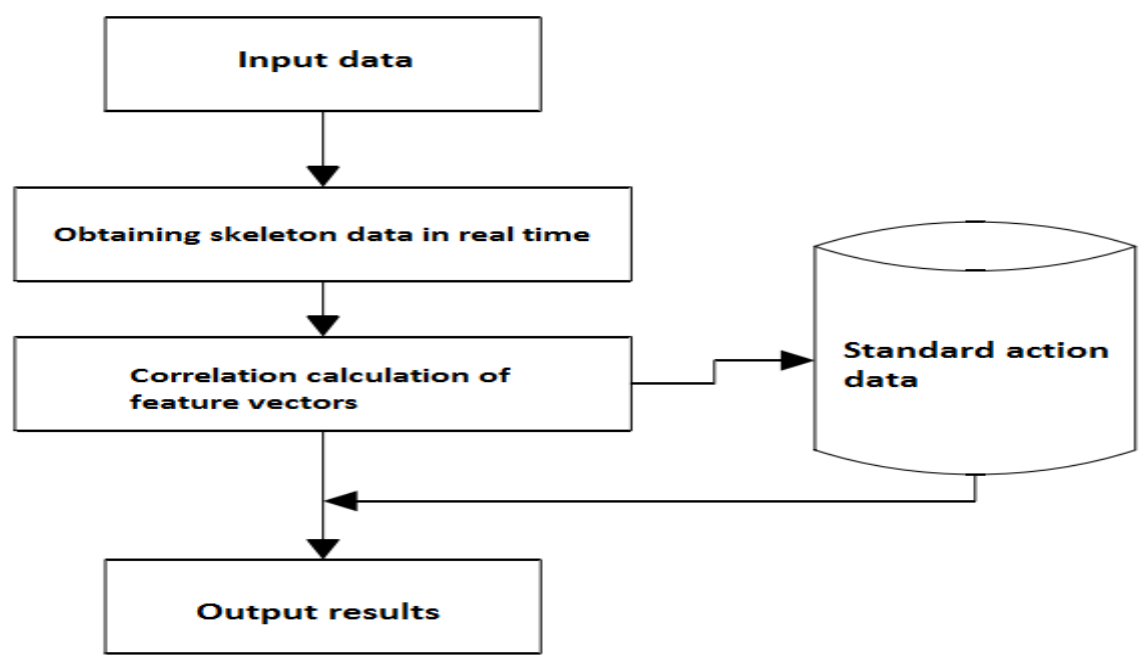

Figure 7. Flow chart of human motion posture analysis method 


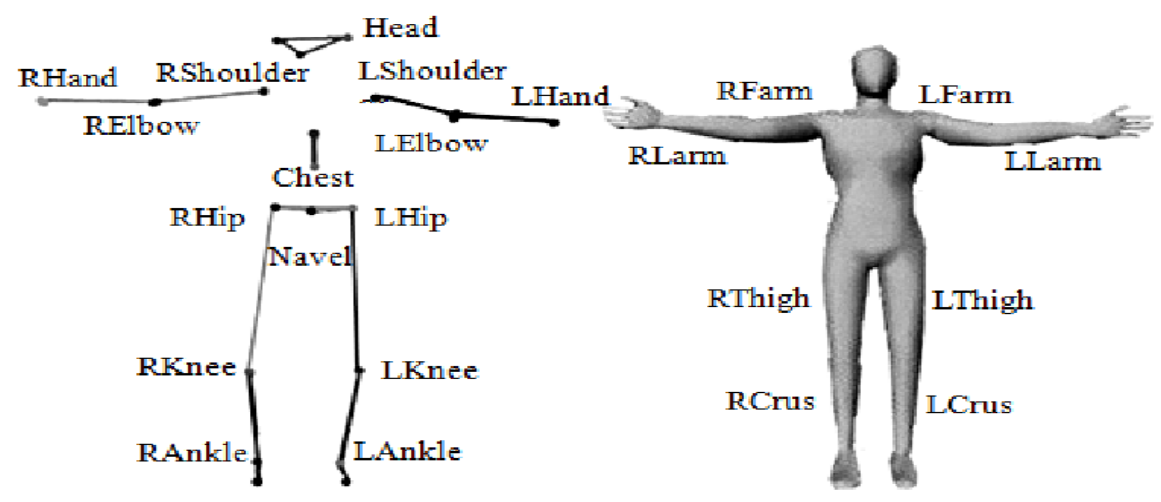

Figure 8. Chain system of human skeleton

The proposed method involves the following steps:

Step 1 Real-time acquisition of skeleton data: obtain the sequence of dance postures in real time through optical motion capture, and save the coordinates of the markers on the human body model in the spatial coordinate system.

Step 2 Posture analysis: Determine 7 characteristic planes according to the feature points, extract the angle between the eigenvectors and the posture eigenvectors, and calculate the feature correlation coefficients of human postures according to the motion features of the key parts.

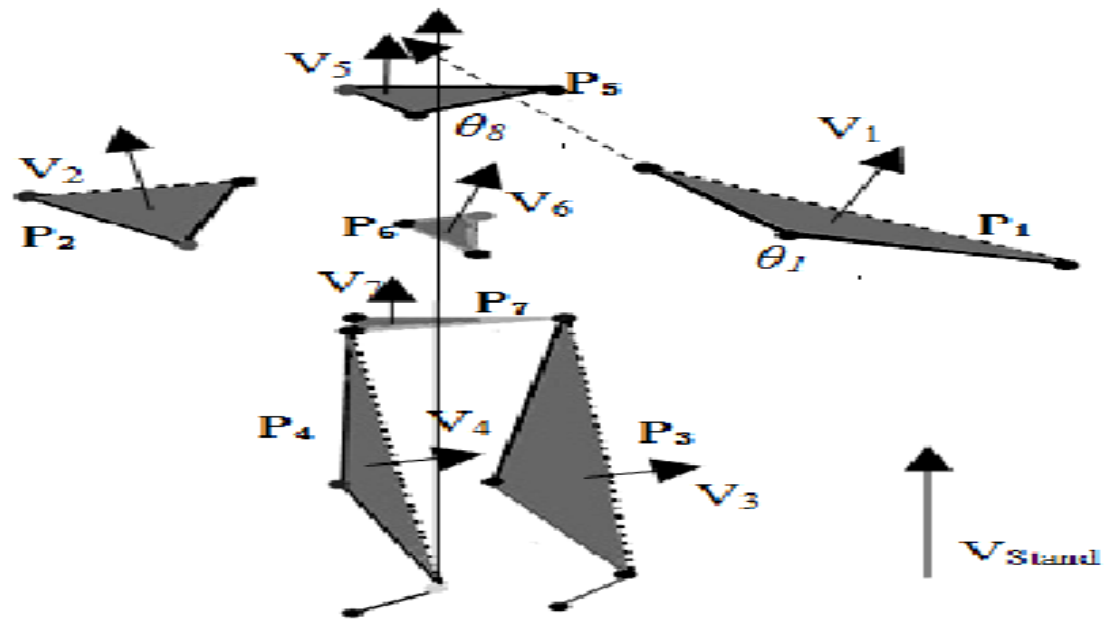

Figure 9. Characteristic planes of human skeleton 
Step 3 Differentiation between feature postures: Identify the difference and accuracy of dance postures and standard motions in light of the correlation coefficients of eigenvectors and their angles.

Without considering muscles and the nervous system, the complex motions of the human skeleton were abstracted into a simple chain system of several rigid bodies (Figure 8).

Following the proposed method, the human skeleton model was extracted by the motion capture system. Since a characteristic plane is determined by 3 feature points, seven characteristic planes were identified based on the 21 markers on the human skeleton for motion postures (Figure 9). Each characteristic plane represents a part of the human body and serves as a basic calculation plane.

Tables 1 and 2 respectively define the eigenvectors and their angles of the main parts in the human body according to the standard dance postures and the relative range of motions of the human skeleton.

Table 1

Discriminant Vectors on Characteristic Planes

\begin{tabular}{lc}
\hline Feature plane & Feature vector \\
\hline Left arm $\left(\mathrm{P}_{1}\right)$ & $\boldsymbol{V}_{\mathbf{1}}=\boldsymbol{V}_{\text {LLarm }} \times \boldsymbol{V}_{\text {LFarm }}$ \\
Right arm $\left(\mathrm{P}_{2}\right)$ & $\boldsymbol{V}_{\mathbf{2}}=\boldsymbol{V}_{\text {RLarm }} \times \boldsymbol{V}_{\text {RFarm }}$ \\
Left leg $\left(\mathrm{P}_{3}\right)$ & $\boldsymbol{V}_{\mathbf{3}}=\boldsymbol{V}_{\text {LThigh }} \times \boldsymbol{V}_{\text {LCrus }}$ \\
Right leg $\left(\mathrm{P}_{4}\right)$ & $\boldsymbol{V}_{\mathbf{4}}=\boldsymbol{V}_{\text {RThigh }} \times \boldsymbol{V}_{\text {RCrus }}$ \\
Head $\left(\mathrm{P}_{5}\right)$ & $\boldsymbol{V}_{\mathbf{5}}=\boldsymbol{V}_{\text {LHead }} \times \boldsymbol{V}_{\text {RHead }}$ \\
Chest $\left(\mathrm{P}_{6}\right)$ & $\boldsymbol{V}_{\mathbf{6}}=\boldsymbol{V}_{\text {LChest }} \times \boldsymbol{V}_{\text {RChest }}$ \\
Hip $\left(\mathrm{P}_{7}\right)$ & $\boldsymbol{V}_{\mathbf{7}}=\boldsymbol{V}_{\text {LHip }} \times \boldsymbol{V}_{\text {RHip }}$ \\
\hline
\end{tabular}

Table 2

Discriminant Angles on Characteristic Planes

\begin{tabular}{lccc}
\hline Feature plane & Geometrical relation & $\boldsymbol{\theta}_{\max }$ & $\boldsymbol{\theta}_{\min }$ \\
\hline Left arm $\left(\mathrm{P}_{1}\right)$ & $\theta_{1}=\left\langle V_{\text {LFarm }}, V_{\text {LLarm }}\right\rangle$ & $180^{\circ}$ & $\mathbf{4 0}^{\circ}$ \\
Right arm $\left(\mathrm{P}_{2}\right)$ & $\theta_{2}=\left\langle V_{\text {RFarm }}, V_{\text {RLarm }}\right\rangle$ & $180^{\circ}$ & $\mathbf{4 0}^{\circ}$ \\
Left leg $\left(\mathrm{P}_{3}\right)$ & $\theta_{3}=\left\langle V_{\text {LCrus }}, V_{\text {LThigh }}\right\rangle$ & $180^{\circ}$ & $\mathbf{4 0}^{\circ}$ \\
Right leg $\left(\mathrm{P}_{4}\right)$ & $\theta_{4}=\left\langle V_{\text {RCrus }}, V_{\text {RThigh }}\right\rangle$ & $180^{\circ}$ & $\mathbf{4 0}^{\circ}$ \\
Head $\left(\mathrm{P}_{5}\right)$ & $\theta_{5}=\left\langle V_{\text {RHead }}, V_{\text {LHead }}\right\rangle$ & - & - \\
Chest $\left(\mathrm{P}_{6}\right)$ & $\theta_{6}=\left\langle V_{\text {RChest }}, V_{\text {LChest }}\right\rangle$ & - & - \\
Hip $\left(\mathrm{P}_{7}\right)$ & $\boldsymbol{\theta}_{\mathbf{7}}=\left\langle\boldsymbol{V}_{\text {RHip }}, \boldsymbol{V}_{\text {LHip }}\right\rangle$ & - & - \\
\hline
\end{tabular}

\section{Digital dance teaching method based on motion capture technology}

Considering the complexity of dance learning, the author developed a dance teaching method based on motion capture technology, which divides a complex dance posture into multiple segments. These segments are easy to be adapted into teaching animation.

\section{Overview of the method}

The motion capture technology was applied to routine dance teaching through the procedure in Figure 10. Firstly, the dancer's motions and their ranges were initialized; then, the dancer's motions were captured and 
converted into 3D data, and a 3D dance teaching database was set up; finally, the database was made into a 3D animation for teaching practice.

In addition, this paper establishes a realistic motion model for dance teaching through the following steps: Firstly, the motion data, which drives the movement of the skeleton model, was matched with markers in the data editor; then, the skeleton model was matched with the standard dance posture models, such that the dancer's motions are consistent with the standard motions. As shown in Figure 11, the motions are adapted into a teaching animation for the students to learn.

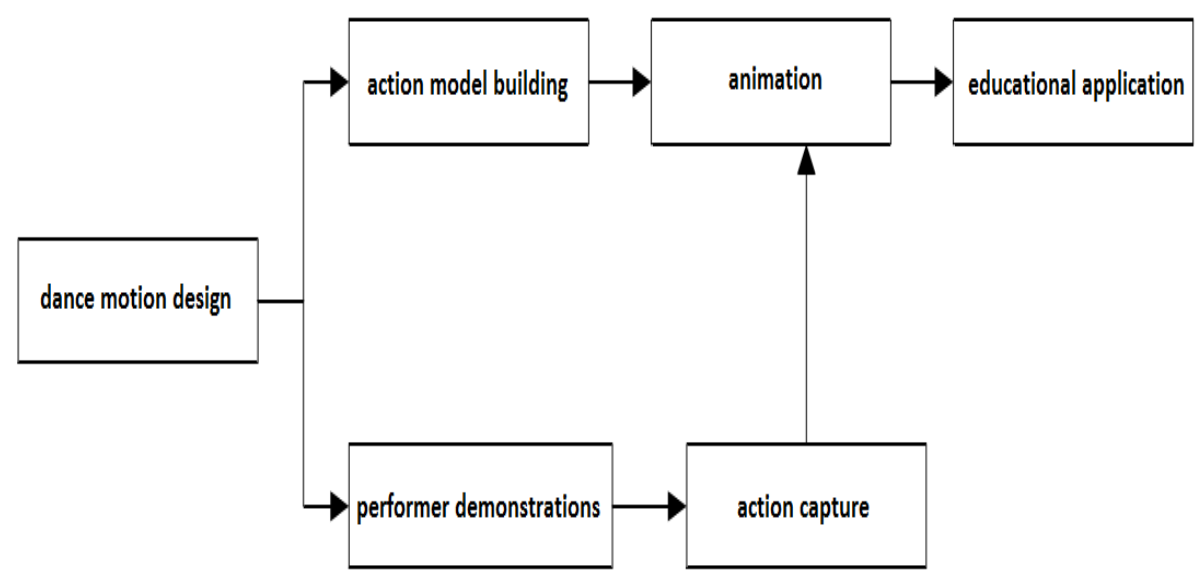

Figure 10. Application of motion capture technology in dance teaching

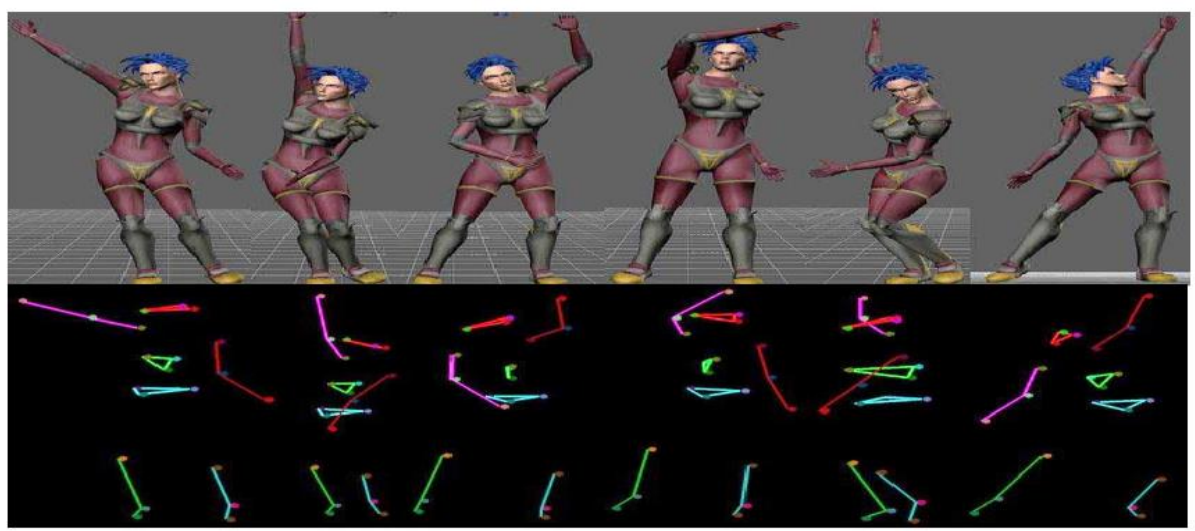

Figure 11. Production of the teaching animation

In this way, the teacher can correct and instruct the motions of each student according to his/her learning progress. The proposed capture system also makes it possible to capture the dance postures of the teacher and the students. The accuracy of real-time detection is guaranteed by the processing of effective 3D data in computer. 


\section{Verification experiment}

A contrastive experiment was performed to verify the effect of the proposed capture technology in dance teaching. A total of 60 students were divided into a control group and a test group by drawing lots. All of them filled out a questionnaire on their motivation, initiative, interest, mastery of basic postures, physical fitness and learning efficiency of dancing. The results indicate no significant difference $(\mathrm{P}>0.05)$ for the two groups of students in these areas. Besides, the students received a test on their theoretical knowledge in dancing. The results reveal that there was no significant difference $(\mathrm{P}>0.05)$ between the two groups. Thus, the subjects are representative and suitable for further experiments.

The students in the control group learned dancing outdoor. They followed the sequence of postures demonstrated by the teacher, who explained the skills to the students and corrected their mistakes. By contrast, those in the test group went to a lab to watch the $3 \mathrm{D}$ animation produced by motion capture, and learn dancing under the guidance of the proposed system. Their learning data was collected and processed by the proposed system, so that their learning situation could be acquired and displayed in real time and their mistakes corrected in time.

After all the teaching hours, the learning situations of the two groups were verified by means of examination. The teacher scored the amplitude, intensity, coherence and technical specificity of the postures and recorded the results in Table 3.

Table 3

Examination Results

\begin{tabular}{lcccc}
\hline Group & Action amplitude & Action strength & Action coherent & Action normative \\
\hline Control group & $80.32 \mp 6.3$ & $78.52 \mp 7.3$ & $81.56 \mp 7.6$ & $77.61 \mp 7.5$ \\
Experimental group & $80.96 \mp 6.7$ & $82.17 \mp 5.8$ & $83.82 \mp 3.1$ & $83.18 \mp 3.2$ \\
Significance & .032 & .028 & .021 & .039 \\
\hline
\end{tabular}

The examination results prove that the students who learned dance postures with the aid of the motion capture system outperformed those in the control group.

\section{Conclusion}

This paper analyses human motion postures based on the real-time features of optical motion capture system, and proposes a posture analysis method in light of eigenvector matching. The motion capture technology was applied to assist digital dance teaching. In this way, the students' learning progress could be quantified, and the wrong dance postures could be corrected in time. Considering the complexity of dance learning, the proposed system was adopted to acquire various postures of dance learners, and contrast their dance postures against the standard ones. The proposed analysis method was verified through a contrastive experiment, shedding new light on the innovation of digital dance teaching. 


\section{References}

Bharatkumar, A. G., Daigle, K. E., Pandy, M. G., \& Cai, Q. (1994). Lower limb kinematics of human walking with the medial axis transformation, Proceedings of the 1994 IEEE Workshop on Motion of Non-Rigid and Articulated Objects, 70-76. https://doi.org/10.1109/MNRAO.1994.346252

Chen, Z., \& Lee, H. J. (2015). Knowledge-guided visual perception of 3D human gait from a single image sequence. IEEE Transactions on Systems Man and Cybernetics, 22(2), 336-342. https://doi.org/10.1109/21.148408

Li, Y., Sun, Z. X., \& Chen, S. L. (2012). 3D Human Pose Analysis from Monocular Video by Simulated Annealed Particle Swarm Optimization. Acta Automatica Sinica, 38(5), 732-741. https://doi.org/10.3724/SP.J.1004.2012.00732\}

Lv, D. S., \& Guo, C. (2011). Digital protection and cultural living space of musical dance performances in confucius memorial ceremony. Journal of Harbin Institute of Technology, 13(5), 99-103.

Moeslund, T. B., \& Granum, E. (2001). A survey of computer vision-based human motion capture. Computer Vision \& Image Understanding, 81(3), 231-268. https://doi.org/10.1006/cviu.2000.0897

Qiu, W. B., \& Li, C. (2009). The research of protection methods about the dances artistic of national minorities based on motion capture technology. Journal of Beijing Dance Academy, 11(4), 101-104.

Renna, I., Chellali, R., \& Achard, C. (2011). Real and simulated upper body tracking with annealing particle filter and belief propagation for human-robot interaction. International Journal of Humanoid Robotics, 8(1), 127-146. https://doi.org/10.1142/S0219843611002368\}

Shinoda, Y., Mito, Y., Ozawa, T., Mizutani, Y., Watanuma, R., \& Marumo, M. (2012). Consideration of classification of dance movements for Nihon Buyo using motion capture system, Sice Conference. IEEE, 1025-1028.

Windsor, B. (2004). Digital Puppetry through Motion Capture. Puppetry Journal.

Yang, J. C., Chen, C. H., \& Ming, C. J. (2010). Integrating video-capture virtual reality technology into a physically interactive learning environment for English learning. Computers \& Education, 55(3), 13461356. https://doi.org/10.1016/j.compedu.2010.06.005

Zhao, Z. X., Dai, H., Zhao, W. B., \& Yuan, J. (2012). Human motion posture simulation based on inertial motion Capture. Computer Engineering, 38(5), 5-8. 